\title{
The metabolism of neuropeptides
}

\section{Phase separation of synaptic membrane preparations with Triton X-114 reveals the presence of aminopeptidase $\mathbf{N}$}

\author{
Rebecca MATSAS,* Sally L. STEPHENSON, John HRYSZKO, A. John KENNY and Anthony J. TURNER $\dagger$ \\ MRC Membrane Peptidase Research Group, Department of Biochemistry, University of Leeds, Leeds LS2 9JT, U.K.
}

\begin{abstract}
The property of solutions of Triton X-114 to separate into detergent-rich and detergent-poor phases at $30^{\circ} \mathrm{C}$ has been exploited to investigate the identities of the aminopeptidases in synaptic membrane preparations from pig striatum. When titrated with an antiserum to aminopeptidase $\mathrm{N}$ (EC 3.4.11.2), synaptic membranes solubilized with Triton X-100 revealed that this enzyme apparently comprises no more than $5 \%$ of the activity releasing tyrosine from [Leu]enkephalin. When assayed in the presence of puromycin, this proportion increased to $20 \%$. Three integral membrane proteins were fractionated by phase separation in Triton X-114. Aminopeptidase activity, endopeptidase-24.11 and peptidyl dipeptidase A partitioned predominantly into the detergent-rich phase when kidney microvillar membranes were so treated. However, only $5.5 \%$ of synaptic membrane aminopeptidase activity partitioned into this phase, although the other peptidases behaved predictably. About half of the aminopeptidase activity in the detergent-rich phase could now be titrated with the antiserum, showing that aminopeptidase $\mathbf{N}$ is an integral membrane protein of this preparation. Three aminopeptidase inhibitors were investigated for their ability to discriminate between the different activities revealed by these experiments. Although amastatin was the most potent $\left(\mathrm{IC}_{50}=5 \times 10^{-7} \mathrm{M}\right)$ it failed to discriminate between pure kidney aminopeptidase $\mathrm{N}$, the total activity of solubilized synaptic membranes and that in the Triton X-114-rich phase. Bestatin was slightly more potent for total activity $\left(\mathrm{IC}_{50}=6.3 \times 10^{-6} \mathrm{M}\right)$ than for the other two forms $\left(\mathrm{IC}_{50}=1.6 \times 10^{-5} \mathrm{M}\right)$. Puromycin was a weak inhibitor, but was more selective. The activity of solubilized membranes was more sensitive $\left(\mathrm{IC}_{50}=1.6 \times 10^{-5} \mathrm{M}\right)$ than that of the pure enzyme or the Triton X-114-rich phase $\left(\mathrm{IC}_{50}=4 \times 10^{-4} \mathrm{M}\right)$. We suggest that the puromycin-sensitive aminopeptidase activity that predominates in crude synaptic membrane preparations may be a cytosolic contaminant or peripheral membrane protein rather than an integral membrane component. Aminopeptidase $\mathrm{N}$ may contribute to the extracellular metabolism of enkephalin and other susceptible neuropeptides in the brain.
\end{abstract}

\section{INTRODUCTION}

A major metabolite of [Met]enkephalin (Tyr-Gly-GlyPhe-Met) in the perfused rat brain in vivo is Tyr-Gly-Gly (Graves et al., 1978). Subsequent studies in vitro have confirmed that membrane preparations from the striatum, a region rich in enkephalin nerve terminals, contain an activity, misleadingly termed 'enkephalinase', that is capable of hydrolysing the $\mathrm{Gly}^{3}-\mathrm{Phe}^{4}$ bond of enkephalin (Malfroy et al., 1978). This activity, originally attributed to the action of angiotensin converting enzyme (peptidyl dipeptidase A; EC 3.4.15.1) (Swerts et al., 1979; Benuck \& Marks, 1979) is now known to be identical with endopeptidase-24.11 (Matsas et al., 1983; Relton et al., 1983) a widely distributed membrane enzyme that is particularly abundant in kidney microvilli. We have suggested that this enzyme plays a general role in the hydrolysis of biologically active peptides including the enkephalins (Matsas et al., 1983).

Inactivation of the enkephalins can also occur through hydrolysis of the $\mathrm{Tyr}^{1}-\mathrm{Gly}^{2}$ bond by aminopeptidases (Hambrook et al., 1976; Meek et al., 1977) and aminopeptidase activity substantially exceeds that of endopeptidase-24.11 in all brain regions examined
(Hudgin et al., 1981; R. Matsas, A. J. Kenny \& A. J. Turner, unpublished work). Hence it is likely that the combined attack of both aminopeptidase and endopeptidase-24.11 is responsible for the extracellular metabolism of enkephalins. Some support for this contention comes from the observation that inhibition of both endopeptidase-24.11 (with thiorphan) and aminopeptidases (with bestatin) provides greater potentiation of enkephalininduced analgesia than is produced by inhibition of either enzyme alone (de la Baume et al., 1982). Furthermore, a combination of bestatin with thiorphan provides maximal protection from metabolism of endogenous enkephalin released from brain slices (de la Baume et al., 1982).

Much of the aminopeptidase activity in brain is cytosolic and therefore not relevant to the synaptic metabolism of the enkephalins (Lane et al., 1977). However, significant quantities of membrane-bound aminopeptidase activity have also been reported to occur in brain (Lane et al., 1977; Knight \& Klee, 1978; Gorenstein \& Snyder, 1979), but the nature of the association of these enzymes with the membrane and their relation to well-characterized aminopeptidases from other tissues remains unclear. A potential candidate is aminopeptidase $\mathrm{N}$ (EC 3.4.11.2) which occurs in the

\footnotetext{
* Present address: Hellenic Pasteur Institute, 127 Vassilissis Sophias Avenue, Athens 11521, Greece.

$\dagger$ To whom correspondence should be addressed.
}

Vol. 231 
kidney microvillar membrane as an integral glycoprotein with its active site facing extracellularly [i.e. an 'ectoenzyme' in the terminology of Engelhardt (1957)]. Thus our immunological approach (Matsas et al., 1983; Relton et al., 1983), using antibodies developed to renal peptidase antigens for characterizing central nervous system peptidases, provides a means for studying brain membrane aminopeptidases and their role in the hydrolysis of enkephalins and other susceptible neuropeptides.

Hersh (1981) has described two membrane enzymes from bovine brain able to cleave the $\mathrm{Tyr}^{1}-\mathrm{Gly}^{2}$ bond of enkephalin. One of these (designated M2) had many properties in common with cytosolic aminopeptidase (e.g. similar $K_{\mathrm{m}}$ for enkephalin and sensitivity to inhibition by puromycin). The other enzyme (M1) was distinct in properties from cytosolic aminopeptidase and had some characteristics in common with aminopeptidase $\mathbf{N}$. However, it was not possible to confirm its identity unambiguously. Hui et al. (1983) have also purified an 'enkephalin aminopeptidase' from rat brain membranes but its possible identity with aminopeptidase $\mathrm{N}$ was not established. Unlike endopeptidase-24.11, which occurs predominantly in the plasma membrane, and which is uniquely inhibited by phosphoramidon, aminopeptidases comprise a family of enzymes that occur in multiple cell locations, and available inhibitors (e.g. puromycin, bestatin, amastatin) show overlapping specificity between different aminopeptidases. Thus, establishing the genuine membrane location of a particular activity requires special caution. In the present paper we have used phase separation with Triton X-114, a technique developed by Bordier (1981) for identifying integral membrane proteins, to show that aminopeptidase $\mathrm{N}$ is a major component of synaptic membrane preparations. Furthermore, the predominant 'puromycin-sensitive' aminopeptidase activity present in such membrane preparations is likely to be an associated peripheral membrane protein or a cytosolic contaminant.

\section{EXPERIMENTAL}

\section{Materials}

[Leu $\left.{ }^{5}\right]$ Enkephalin was obtained from Cambridge Research Biochemicals (Harston, Cambridge, U.K.). HipHis-Leu, puromycin, bestatin, amastatin and Triton X-114 were from Sigma Chemical Co. The Triton X-114 was subjected to precondensation before use, as described by Bordier (1981). The antiserum to pig kidney aminopeptidase $\mathbf{N}$ was a gift from Dr. M. Danielsen, Copenhagen. Other materials were from sources previously noted (Matsas et al., 1983, 1984a,b, 1985; Relton et al., 1983).

\section{Methods}

Preparation of membrane fractions. Kidney microvillar membranes were prepared as described by Booth \& Kenny (1974). Synaptic membranes were prepared from pig striatum as in Matsas et al. (1983) with the exception that, in some preparations, a cocktail of proteinase inhibitors consisting of di-isopropylfluorophosphate $(0.1 \mathrm{mM})$, antipain $(1 \mu \mathrm{g} / \mathrm{ml})$, leupeptin $(1 \mu \mathrm{g} / \mathrm{ml})$ and pepstatin $(1 \mu \mathrm{g} / \mathrm{ml})$ was included in the homogenization buffer. The specific activity of lactate dehydrogenase (EC 1.1.1.27) in the washed synaptic membrane fraction was 0.07 relative to the original homogenate.
Solubilization and phase separation of membrane fractions with Triton X-114. This was performed as described by Bordier (1981). Briefly, a 7:1 (w/w) ratio of detergent:protein was used and solubilization was carried out for $1 \mathrm{~h}$ at $4^{\circ} \mathrm{C}$. After precipitation of unsolubilized material $\left(30000 \mathrm{~g}, 1 \mathrm{~h}\right.$ at $\left.4{ }^{\circ} \mathrm{C}\right)$, the supernatant was warmed up to $30^{\circ} \mathrm{C}$ and phase separation was completed by centrifugation at $3000 \mathrm{~g}$ for $5 \mathrm{~min}$ at $30^{\circ} \mathrm{C}$. The detergent-poor phase was removed by using a Pasteur pipette and the detergent-rich phase was washed and re-extracted as above.

Assay of aminopeptidase activity releasing tyrosine from [Leu ${ }^{5}$ ]enkephalin. Samples of synaptic membranes $(50 \mu \mathrm{g}$, 15 min incubation) or kidney microvillar membranes $(0.5 \mu \mathrm{g}, 15 \mathrm{~min}$ incubation) in $0.1 \mathrm{M}$ Tris $/ \mathrm{HCl}$ at $\mathrm{pH} 7.4$ were incubated with [Leu $]$ enkephalin $(0.5$ or $1 \mathrm{~mm})$ at $37^{\circ} \mathrm{C}$ (final volume $100 \mu \mathrm{l}$ ) in the presence of $1 \mu \mathrm{M}$ phosphoramidon and $1 \mu \mathrm{M}$-captopril to inhibit endopeptidase-24.11 and peptidyl dipeptidase A activity, respectively. The reaction was stopped by immersing in a boiling-water bath for $4 \mathrm{~min}$ and protein was removed by centrifugation. The release of tyrosine was then monitored by h.p.l.c. analysis on a linear gradient of acetonitrile $(4.5 \%-30 \%)$ as described in Matsas et al. (1983). Under these conditions, tyrosine-was eluted at $3.4 \mathrm{~min}$. Inhibitors were added to the incubation mixture before the substrate, but no preincubation was used.

Assay of endopeptidase-24.11. [D-Ala $\left.{ }^{2}, \mathrm{Leu}^{5}\right]$ Enkephalin was used as substrate in the presence and absence of $1 \mu \mathrm{M}$-phosphoramidon as described in Matsas et al. (1983).

Assay of peptidyl dipeptidase A. Hip-His-Leu was used as substrate and the product (hippurate) was quantified by h.p.1.c. (Matsas et al., 1984a).

Purification of aminopeptidase $\mathbf{N}$. This was purified to homogeneity from pig kidney as described by George \& Kenny (1973) except that the preparative electrophoresis step was replaced by gel filtration on Sepharose 6B and a second DEAE step. The specific activity of the final product was $19.4 \mu \mathrm{mol} / \mathrm{min}$ per $\mathrm{mg}$ of protein assayed with L-alanine-4-methyl-7-coumarylamide as substrate (Fulcher \& Kenny, 1983).

\section{RESULTS}

Immunotitration of aminopeptidase activity from synaptic membranes and kidney microvillar membranes

Membrane fractions, solubilized with Triton X-100, were titrated with antisera raised to kidney aminopeptidase $\mathrm{N}$. The hydrolysis of the $\mathrm{Tyr}^{1}-\mathrm{Gly}^{2}$ bond of enkephalin by kidney microvillar membranes was substantially inhibited $(78 \%$ ) by the antiserum (Fig. 1a). However, hydrolysis by the synaptic membranes under identical conditions was inhibited by less than $5 \%$, initially suggesting that aminopeptidase $\mathrm{N}$, if present at all in synaptic membranes, represented a relatively minor component of the fraction (Fig. 1a). Preimmune serum showed no inhibitory effect. When the immunotitrations were repeated in the presence of $0.1 \mathrm{~mm}$-puromycin, substantially greater inhibition of aminopeptidase activity was achieved. In the case of kidney membranes, the antiserum totally abolished the hydrolysis of the 
(a)

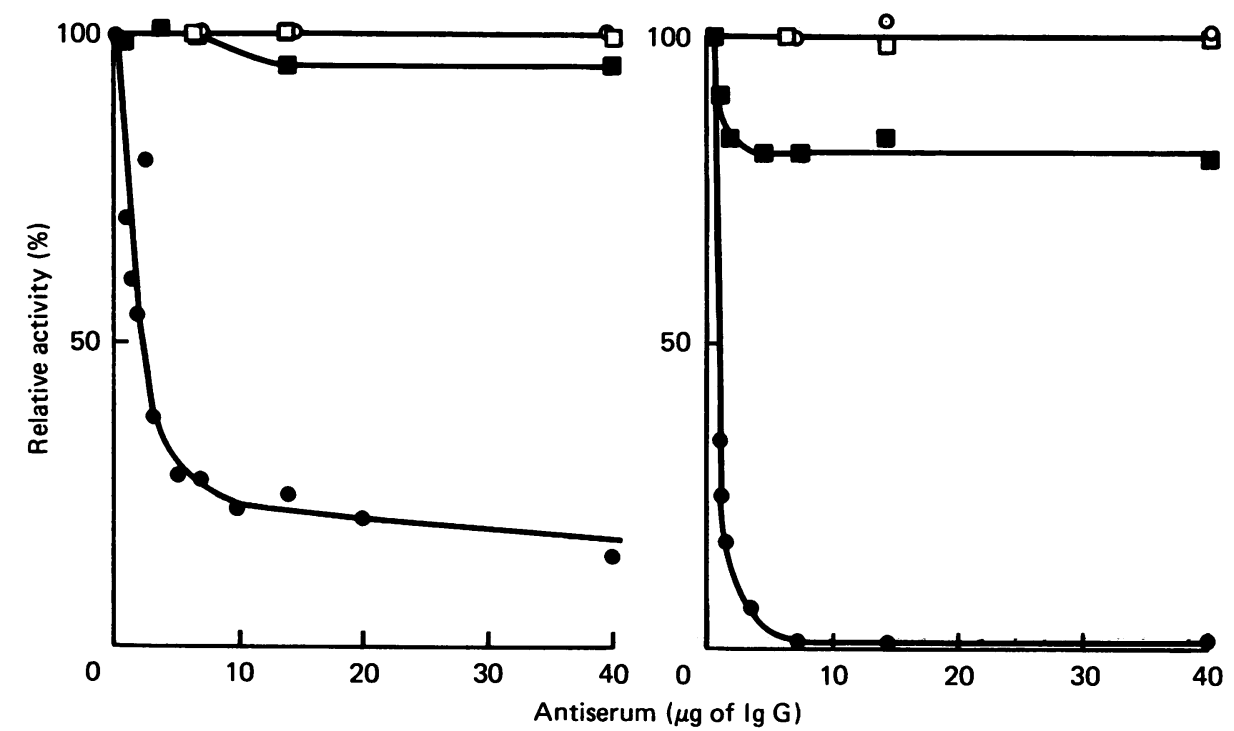

Fig. 1. Inhibition of aminopeptidase activity in striatal synaptic membranes and kidney microvilli by antiserum raised to kidney aminopeptidase $\mathbf{N}$

$\left[\mathrm{Leu}^{5}\right]$ Enkephalin was used as substrate and the release of tyrosine was monitored by h.p.l.c. (a) Membranes solubilized with Triton X-100; $(b)$ membranes solubilized with Triton X-100 and assayed in the presence of 0.1 mM-puromycin. Open symbols correspond to preimmune serum, closed symbols to immune serum. $\square, \square$, Synaptic membranes;,$\bigcirc$, microvillar membranes.

$\mathrm{Tyr}^{1}-\mathrm{Gly}^{2}$ bond of enkephalin and, in the case of synaptic membranes, $20 \%$ inhibition was achieved. These data suggested the presence of a 'puromycin-sensitive' aminopeptidase as a relatively minor component of total aminopeptidase activity in kidney membrane fractions but a major component in the synaptic membrane preparation.

\section{Triton X-114 extraction of membrane peptidases}

In order to investigate whether the "puromycinsensitive' aminopeptidase was an integral component of synaptic membranes, extraction of the membrane preparations with the detergent Triton. X-114 at $30^{\circ} \mathrm{C}$ was employed. Under the conditions used, integral membrane proteins are recovered in the detergent-rich phase when phase separation occurs, whereas hydrophilic, peripheral or cytosolic proteins remain in the detergentpoor phase (Bordier, 1981).
Table 1 demonstrates that endopeptidase-24.11 and peptidyl dipeptidase $A$, both well established as integral membrane proteins, extracted predominantly into the detergent-rich phase after phase separation of either kidney microvillar or synaptic membranes. However, the distribution of aminopeptidase activity (measured by the release of tyrosine from [Leu]enkephalin) differed markedly between kidney and brain membrane preparations. Much of the kidney aminopeptidase $(62 \%)$ appeared in the detergent-rich phase, consistent with its identity as aminopeptidase $\mathbf{N}$. However, relatively little $(5.5 \%)$ of the brain membrane aminopeptidase activity extracted into the detergent-rich phase. The same results were obtained with synaptic membranes prepared in the presence of proteinase inhibitors. Thus, the bulk of the activity releasing tyrosine from enkephalin that was observed in synaptic membrane preparations had hydrophilic characteristics and may represent adherent

\section{Table 1. Distribution of peptidase activities following phase separation of membrane proteins with Triton X-114}

Membrane preparations were treated with Triton X-114 and subjected to phase separation as described in the Methods section. The results are expressed as precentage of enzyme activity recovered in the detergent and aqueous phases. The overall recovery for all the enzymes was in the range $60-70 \%$. The results are the means for two experiments.

\begin{tabular}{|c|c|c|c|}
\hline \multirow[b]{2}{*}{ Membrane fraction } & \multirow[b]{2}{*}{ Enzyme } & \multicolumn{2}{|c|}{ Distribution ( $\%$ ) } \\
\hline & & Detergent-rich phase & Detergent-poor phase \\
\hline $\begin{array}{l}\text { Kidney microvillar } \\
\text { membranes }\end{array}$ & $\begin{array}{l}\text { Aminopeptidase } \mathrm{N} \\
\text { Endopeptidase-24.11 } \\
\text { Peptidyl dipeptidase A }\end{array}$ & $\begin{array}{l}62 \\
82 \\
95\end{array}$ & $\begin{array}{r}38 \\
18 \\
5\end{array}$ \\
\hline Synaptic membranes & $\begin{array}{l}\text { Aminopeptidase } \\
\text { Endopeptidase-24.11 } \\
\text { Peptidyl dipeptidase A }\end{array}$ & $\begin{array}{l}5.5 \\
96 \\
90\end{array}$ & $\begin{array}{c}94.5 \\
4 \\
10\end{array}$ \\
\hline
\end{tabular}


cytosolic enzyme or a peripheral membrane aminopeptidase that was associated electrostatically with the membrane. The characteristics of the aminopeptidase activities that extract into the detergent-rich and detergent-poor phases were investigated further.

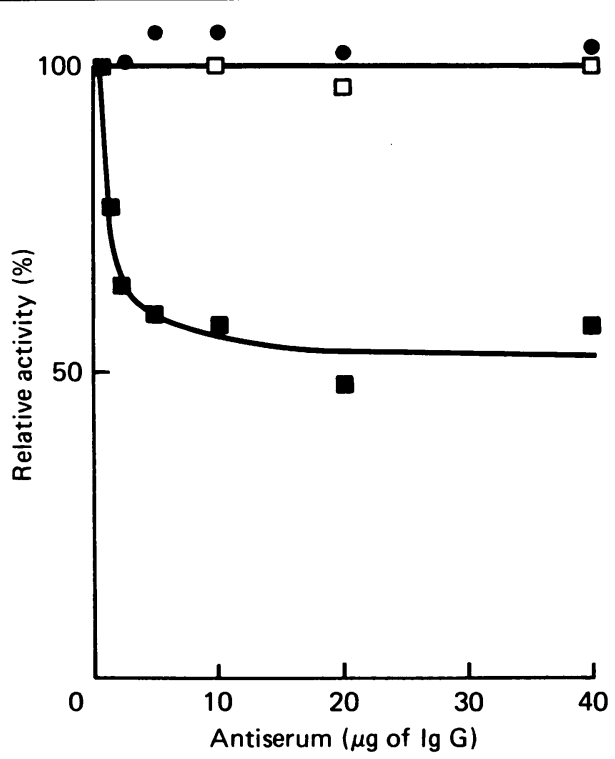

Fig. 2. Inhibition of aminopeptidase activity in striatal synaptic membranes after phase separation with Triton X-114

[Leu $\left.{ }^{5}\right]$ Enkephalin was used as substrate and the release of tyrosine was monitored by h.p.l.c. The symbols correspond to: $\square$, detergent-rich phase, immune serum; $\square$, detergentrich phase, preimmune serum; $\mathbf{0}$, detergent-poor phase, immune serum.
Immunotitration of synaptic membrane aminopeptidase activities after phase separation with Triton X-114

Synaptic membranes were subjected to phase separation with Triton X-114 and the detergent-rich and detergentpoor phases were separately titrated with the antiserum raised to kidney aminopeptidase N. Substantial inhibition (approx. 50\%) of tyrosine release was now observed with the detergent phase (compare Fig. 2 with Fig. 1a), indicating that aminopeptidase $\mathbf{N}$ is a substantial component of synaptic membranes where it may exist as an ectoenzyme, as is the case for the kidney microvillar membrane (Kenny \& Maroux, 1982). No inhibition of aminopeptidase activity by the antiserum occurred in the detergent-poor phase, suggesting that proteolysis had not released a hydrophilic form of the enzyme. Pre-immune serum was not inhibitory to either phase (Fig. 2).

Inhibition of synaptic membrane aminopeptidase activity present in the Triton $\mathrm{X}-114$ detergent-rich phase

The effects of three aminopeptidase inhibitors (puromycin, bestatin and amastatin) were compared on the hydrolysis of the $\mathrm{Tyr}^{1}-\mathrm{Gly}^{2}$ bond of enkephalin by a homogeneous preparation of kidney aminopeptidase $\mathrm{N}$, by synaptic membranes and by the detergent-rich phase obtained after phase separation of synaptic membranes with Triton X-114. In all cases the most potent inhibitor was amastatin, followed by bestatin and puromycin (Fig. 3 ). The detergent extracts and purified enzyme appeared equally sensitive to amastatin (Fig. $3 a$ ). There was also little difference in sensitivity of the three extracts to bestatin (Fig. 3b). However, puromycin was substantially less potent as an inhibitor of aminopeptidase $\mathrm{N}$ and of the Triton X-114 extract compared with a synaptic membrane preparation before treatment with Triton $\mathrm{X}-114$ (Fig. 3c). These results further suggest that washed (a)

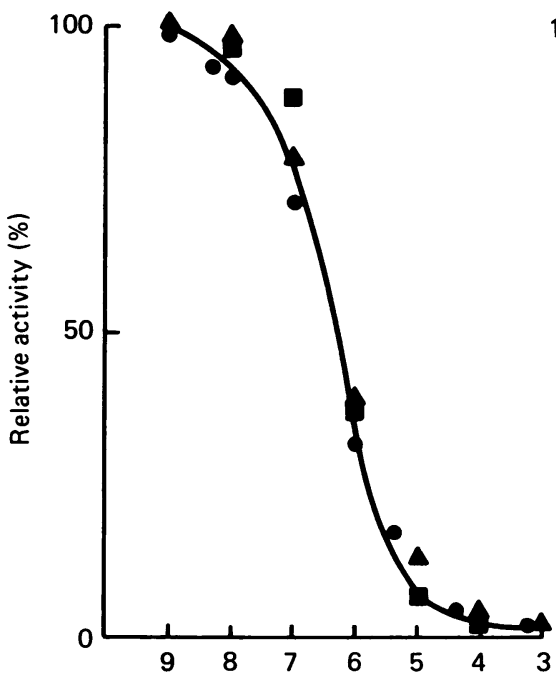

$(b)$

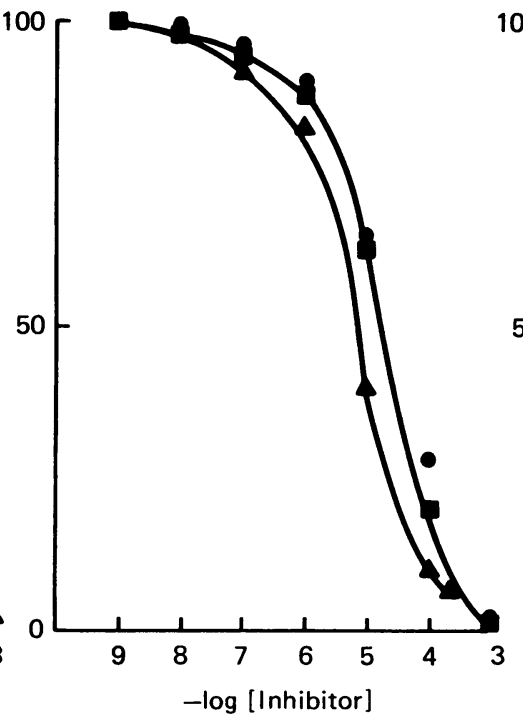

(c)

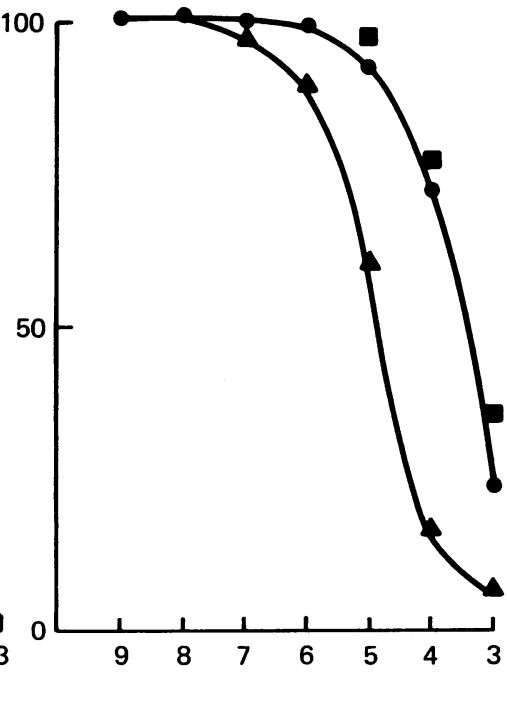

Fig. 3. Inhibition of aminopeptidase activity by amastatin, bestatin and puromycin

[Leu $\left.{ }^{5}\right]$ Enkephalin was used as substrate and the release of tyrosine was monitored by h.p.l.c. (a) Inhibition by amastatin; $(b)$ inhibition by bestatin; $(c)$ inhibition by puromycin. The symbols correspond to: $\boldsymbol{\Delta}$, synaptic membranes solubilized with Triton $\mathrm{X}-100$;, , detergent-rich phase after phase separation of synaptic membranes with Triton X-114; $\square$, purified pig kidney aminopeptidase $\mathbf{N}$. 
synaptic membranes contain substantial quantities of a 'puromycin-sensitive' aminopeptidase that has hydrophilic characteristics and may not represent an integral membrane enzyme. The synaptic membrane aminopeptidase that extracts into the detergent-rich phase with Triton X-114 has immunological and inhibitory characteristics consistent with its identity as aminopeptidase $\mathrm{N}$.

\section{DISCUSSION}

The physiological inactivation of enkephalin at the cell surface involves endopeptidase-24.11 and one or more aminopeptidases. The relative importance of these activities to enkephalin hydrolysis may vary between brain regions, since endopeptidase- 24.11 shows a markedly non-uniform distribution throughout the brain (Matsas et al., 1985). Inhibition of both endopeptidase24.11 (with thiorphan) and aminopeptidases (with bestatin) protects endogenous enkephalin from hydrolysis to a greater extent than either inhibitor alone (de la Baume et al., 1982). However, puromycin, which substantially inhibits aminopeptidase activity in crude brain membrane preparations (Vogel \& Altstein, 1979) has little protective effect on enkephalin released from brain slices (de la Baume et al., 1982). The present data suggest a possible explanation for this anomaly. Extraction of synaptic membranes with Triton X-114 resolves aminopeptidase activity into (i) a 'puromycinsensitive' form that is hydrophilic in character and may correspond to a cytosolic or peripheral membrane enzyme inaccessible to released enkephalin and (ii) an activity that is substantially less sensitive to the action of puromycin. Much of the second activity is immunologically identical with aminopeptidase $\mathrm{N}$, an established plasma membrane ectoenzyme, although the presence of a second membrane aminopeptidase capable of hydrolysing enkephalin cannot be excluded (see Fig. 2).

Two features, therefore, emerge from the present paper. The procedure of phase separation with Triton $\mathrm{X}-114$ provides a valuable extraction procedure for the isolation and characterization of integral membrane peptidases. It is clear that caution should be used in interpreting studies of peptide metabolism that employ crude membrane preparations. Secondly, aminopeptidase $\mathrm{N}$ is a major component of synaptic membranes and may play the predominant role in the release of tyrosine from [Leu]enkephalin at the synapse, as well as in the metabolism of other susceptible neuropeptides such as cholecystokinin-8 (Matsas et al., 1984b). The availability of antibodies, including a monoclonal antibody GK8C1 (N. S. Gee \& A. J. Kenny, unpublished work) to aminopeptidase $\mathrm{N}$ should allow its distribution in the brain to be mapped by immunocytochemical techniques.
Furthermore, kidney aminopeptidase $\mathrm{N}$, which is much more abundant and more easily purified than the brain enzyme, will be useful for studies of the specificity of neuropeptide hydrolysis and the development of more potent and selective inhibitors than either bestatin or amastatin.

We thank the Medical Research Council and Merck, Sharp \& Dohme Research Laboratories for financial support for R.M. S.L.S. is in receipt of an S.E.R.C. CASE Studentship in association with Pfizer Ltd.

\section{REFERENCES}

Benuck, M. \& Marks, N. (1979) Biochem. Biophys. Res. Commun. 88, 215-221

Booth, A. G. \& Kenny, A. J. (1974) Biochem. J. 142, 575-581

Bordier, C. (1981) J. Biol. Chem. 256, 1604-1607

de la Baume, S., Gros, C., Yi, C.-C., Chaillet, P., MarcaisCollado, H., Costentin, J. \& Schwartz, J.-C. (1982) Life Sci. 37, 1753-1756

Engelhardt, W. A. (1957) Proc. Int. Symp. Enzyme Chemistry (Tokyo), I.U.B. Symp. 2, 163-166

Fulcher, I. S. \& Kenny, A. J. (1983) Biochem. J. 211, 743-753

George, S. G. \& Kenny, A. J. (1973) Biochem. J. 134, 43-57

Gorenstein, C. \& Snyder, S. H. (1979) Life Sci. 25, 2065-2070

Graves, F. B., Law, P. Y., Hunt, C. A. \& Loh, H. H. (1978) J. Pharm. Exp. Ther. 206, 492-506

Hambrook, J. M., Morgan, B. A., Rance, M. J. \& Smith, C. F. C. (1976) Nature (London) 262, 782-783

Hersh, L. B. (1981) Biochemistry 20, 2345-2350

Hudgin, R. L., Charleson, S. E., Zimmerman, M., Mumford, R. \& Wood, P. L. (1981) Life Sci. 29, 2593-2601

Hui, K. S., Wang, Y.-J. \& Lajtha, A. (1983) Biochemistry 22, 1062-1067

Kenny, A. J. \& Maroux, S. (1982) Physiol. Rev. 62, 91-128

Knight, M. \& Klee, W. A. (1978) J. Biol. Chem. 253, 3843-3847

Lane, A. C., Rance, M. J. \& Walter, D. S. (1977) Nature (London) 269, 75-76

Malfroy, B., Swerts, J. P., Guyon, A., Roques, B. P. \& Schwartz, J.-C. (1978) Nature (London) 276, 523-526

Matsas, R., Fulcher, I. S., Kenny, A. J. \& Turner, A. J. (1983) Proc. Natl. Acad. Sci. U.S.A. 80, 3111-3115

Matsas, R., Kenny, A. J. \& Turner, A. J. (1984a) Biochem. J. 223, 432-440

Matsas, R., Turner, A. J. \& Kenny, A. J. (1984b) FEBS Lett. 175, 124-128

Matsas, R., Rattray, M., Kenny, A. J. \& Turner, A. J. (1985) Biochem. J. 228, 487-492

Meek, J. L., Yang, H. Y. T. \& Costa, E. (1977) Neuropharmacology 16, 151-154

Relton, J., Gee, N. S., Matsas, R., Turner, A. J. \& Kenny, A. J. (1983) Biochem. J. 215, 519-523

Swerts, J. P., Perdrisot, R., Malfroy, B. \& Schwartz, J. C. (1979)

Eur. J. Pharmacol. 53, 209-210

Vogel, Z. \& Altstein, M. (1979) FEBS Lett. 98, 44-48

Received 20 May 1985; accepted 26 June 1985 